This is the final peer-reviewed accepted manuscript of:

L. Possenti et al., "Transmission loss evaluation for Fabry-Perot materials' characterization," 2020 14th European Conference on Antennas and Propagation (EuCAP), Copenhagen, Denmark, 2020, pp. 1-5

The final published version is available online at:

DOI: https://doi.org/10.23919/EuCAP48036.2020.9136075

Rights / License:

The terms and conditions for the reuse of this version of the manuscript are specified in the publishing policy. For all terms of use and more information see the publisher's website.

This item was downloaded from IRIS Università di Bologna (https://cris.unibo.it/)

When citing, please refer to the published version. 


\title{
Transmission loss evaluation for Fabry-Perot materials' characterization
}

\author{
Leonardo Possenti ${ }^{1}$, Juan Pascual-García ${ }^{2}$, Vittorio Degli-Esposti ${ }^{1}$, Antonio Lozano-Guerrero ${ }^{2}$, Marina Barbiroli ${ }^{1}$, \\ Maria-Teresa Martinez-Inglés ${ }^{3}$, Franco Fuschini ${ }^{1}$, José-Víctor Rodríguez ${ }^{2}$, \\ Enrico Maria Vitucci ${ }^{1}$, José-María Molina-García-Pardo ${ }^{2}$ \\ ${ }^{1}$ Department of Electrical, Electronic and Information Engineering "Guglielmo Marconi” (DEI) University of Bologna, Italy, \\ [leonardo.possenti2,vdegliesposti,marina.barbiroli,franco.fuschini,enricomaria.vitucci]@unibo.it \\ 2Dpto. Tecnologías de la Información y las Comunicaciones, Universidad Politécnica de Cartagena, Spain \\ [Juan.Pascual, Antonio.Lozano,Jvictor.Rodriguez, JoseMaria.Molina]@uptc.es \\ 3University Center of Defense, San Javier, Air Force Base, Ministerio de Defensa, UPCT
}

[mteresa.martinez]@ cud.upct.es

\begin{abstract}
The knowledge of the electromagnetic properties of construction materials is crucial for the design of future wireless systems and for the practical use of deterministic raybased propagation prediction tools. More specifically, with the allocation of several new frequency bands for $5 G$ and beyond systems, the understanding of the electromagnetic properties of materials at those frequencies is needed to determine attenuation and reflectivity of walls and objects. In this work, a wideband method for the characterization of the imaginary part of the complex permittivity is presented; the method is intended to be combined with the recently proposed Fabry-Perot method to measure the permittivity's real part. Paraffin is selected as a reference material since it has long been used to assess methods in the literature.
\end{abstract}

Index Terms- Radio Propagation, Material Characterizacion, 5G, Ray Tracing.

\section{INTRODUCTION}

With the forthcoming allocation of multiple frequency bands for $5 \mathrm{G}$ (and beyond) wireless systems worldwide, including mm-wave frequencies leading to high penetration losses due to walls and objects, simple and fast electromagnetic characterization of construction materials at multiple frequency bands has become an urgent need [1]. Such a characterization is also necessary to use ray-based propagation models, which are increasingly popular for the design of mobile radio systems.

Recently, a simple and fast method for the characterization of construction materials based on the Fabry-Perot (FP) resonance that takes place inside a material slab has been proposed [2]. Such a method easily determines the real part of the permittivity $\left(\varepsilon^{\prime}\right.$, with $\left.\varepsilon=\varepsilon^{\prime}-j \varepsilon^{\prime \prime}\right)$, while its imaginary part $\varepsilon^{\prime \prime}$ is separately determined using a transmission method by trying to match the measured and simulated transmission coefficient over the considered bandwidth. The exact procedure for assessing $\varepsilon^{\prime \prime}$ is not discussed in [2], where the main focus is on the FP measurement principle for the estimation of $\varepsilon^{\prime}$.

Although building materials are low-loss at most frequencies, $\varepsilon^{\prime \prime}$ is very important to determine the overall wall-penetration loss, a critical parameter for the design and deployment of mm-wave systems.

In this present work we define an optimal procedure for the determination of $\varepsilon^{\prime \prime}$ in order to complement the FP procedure shown in [2]. The procedure is based on a leastsquare optimization method and on the use of time-gating [3] to eliminate environment multipath or, if viable case, to cut off the FP resonance from the transmission measurement. Moreover, we discuss the achievable accuracy by comparison with an open-ended coaxial probe-based method [4].

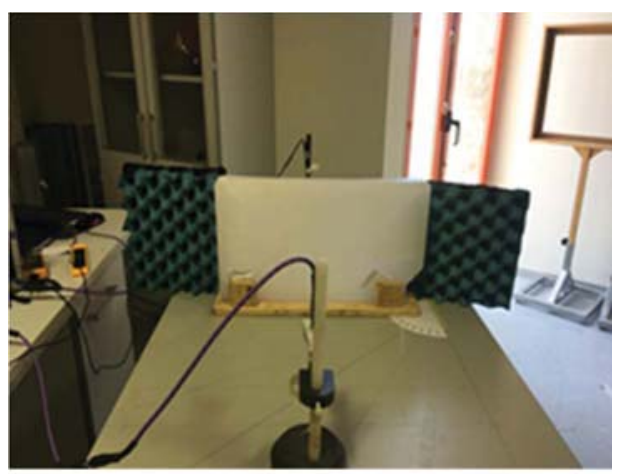

(a)

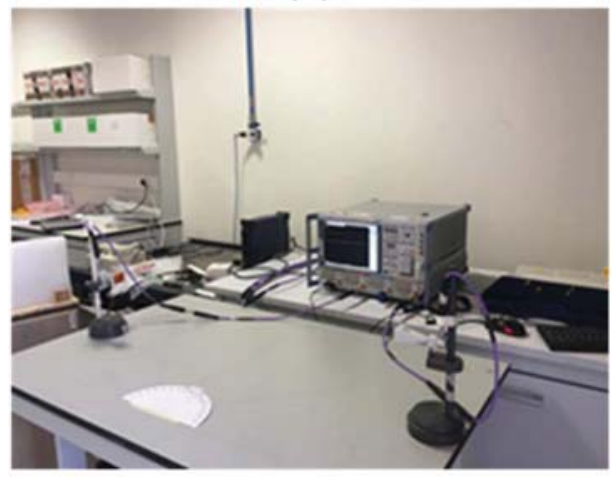

(b)

Fig. 1 - Transmission measurement setup with (a) and without (b) the MUT. 
Since the resonance phenomena that can take place in material slabs is often disregarded when wall-penetration loss measurements are carried out to derive Building Penetration Loss (BPL) models [5] [6], we believe that the present work can be of interest also to foster the realization of future BPL measurement campaigns using a wider bandwidth to account for the frequency-domain ripple due to FP resonance or to periodical structures - e.g. hollow bricks, metal meshes, etc. - that can be present in real building walls and can affect narrow-band measurements.

\section{TRANSMISSION MEASUREMENTS}

In this present work, we considered the $8-14 \mathrm{GHz}$ frequency band as a reference for assessing the presented approach with a well-known method based on probesounding [4].

The considered material under test (MUT) is a uniform flat layer of paraffin with constant thickness. According to previous works [7], [8], paraffin wax shows a very stable value of the permittivity $\left(\varepsilon^{\prime}=2.2-2.3\right)$ over the entire bandwidth, thus it has been chosen as a reference material for assessing $\varepsilon^{\prime \prime}=\sigma / \omega$, being $\sigma$ the material's conductivity $[\mathrm{S} / \mathrm{m}]$, directly or through the tangent $\operatorname{loss}\left(\tan \delta=\varepsilon^{\prime \prime} / \varepsilon^{\prime}\right)$.

\section{A. The measurement setup}

As shown in Fig. 1 the measurements have been performed with a Rodhe\&Schwarz VNA ZVA67, working in the frequency range from $10 \mathrm{MHz}$ to $67 \mathrm{GHz}$. The VNA is linked to the antennas through 50 Ohm ZV-Z193 RF Test Cable. The antennas for the $10 \mathrm{GHz}$ band are two ARRA horn antennas model X820, with $30^{\circ}$ of HPBW and a gain from 15 $\mathrm{dBi}$ to $18 \mathrm{dBi}$ proportional to the frequency.

The antennas have been positioned with their pedestal at a height $20 \mathrm{~cm}$ above the table. In order to ensure far field conditions and the absence of diffraction from the MUT's edges, the distance between the MUT and the antennas has been fixed to $40 \mathrm{~cm}$.

Fig. 1 depicts also the setup for transmission measurement. Two RF absorbers are used to prevent diffracted paths.

\section{B. Transmission loss evaluation procedure}

The measured $\mathrm{S}_{21}$ parameter value is saved into the postprocessing script, then the transmission coefficient is extracted by de-embedding antenna gains and free-space pathloss from the measured $\mathrm{S}_{21}$ parameter. To do so, we remove the MUT and take a free-space LOS snapshot, as shown in Fig. 1 (a).

To compute the transmission losses, that heavily depend on the conductivity $\sigma$ we refer to the model described in [9] which has been modified to consider losses in the MUT and non-orthogonal incidence. We run the model for different values of $\sigma$, and through an optimization algorithm we find the best match in terms of root mean square error (RMSE) between the simulated and measured curves of the absolute value of the transmission coefficient over the considered band, that typically shows a ripple due to Fabry-Perot resonance [2].
The procedure leads to an estimate of the transmission loss of the MUT over the considered bandwidth. As the procedure provides only one value, it has to be considered the mean value for such frequency band.

\section{The time-gating method}

As procedure for the evaluation of $\sigma$ is based on the minimization of the error between model and measurement, it is important to reduce as much as possible every source of noise (i.e. multipath components due to the environment) from the measured transmission coefficient.

We apply the time gating technique to select only the desired component, which is, for transmission measurement, the first-arrival of the impulse response in the time domain, obtained from the Fourier transform of the frequency response.

It is important to assess the width of the considered time window. On the one hand, a very narrow gate could hinder the FP resonance by cutting multiple reflections inside the slab: this could be desirable to select only the forward propagating wave and eliminate the ripple, but we found it would require too large a bandwidth to be feasible. On the other hand, a very large gate could include also undesired multipath components, coming from the surrounding environment. The time gate should include only those waves whose power is above the noise level. In order to select an appropriate time gate, we have estimated the field each one of the transmitted waves seen in Fig. 2. The field of the $\mathrm{N}^{\text {th }}$ transmitted wave is [9]:

$$
\begin{gathered}
E_{T, N}=\frac{1}{d_{t x_{-} r x}} e^{-j k_{1} d_{t x_{-} r x} \cdot T_{1} \cdot T_{2} \cdot \rho_{1}^{(2 N-2)} e^{-(2 N-1) \gamma_{2} L}} \\
\cdot e^{(N-1) j k_{1} d_{e x t r a}} \cdot e^{j k_{1} t}
\end{gathered}
$$

where $T_{1}$ is the transmission coefficient in the air to material interface, $T_{2}$ is the transmission coefficient in the material to air interface, $\rho_{1}$ is the reflection coefficient in the air-material interface, $\gamma_{2}$ is the propagation constant inside the material, $k$ is the wavenumber in the air, $t$ is the difference between $d_{t x \_r x}$ and the distance travelled by the first transmitted wave as defined in [9]. The distance $d_{\text {extra }}$ is:

$$
d_{\text {extra }}=2 L \sin \left(\theta_{i}\right) \sin \left(\theta_{t}\right) .
$$

The angle of transmission $\theta_{t}$ and the distance $L$ depend on the permittivity of the material. Nevertheless, the value estimated from the Fabry-Perot technique could be used in the estimation of the mentioned angles. Once all previous data is calculated the attenuation between the first transmitted wave $\left(E_{t 1}\right)$ and the following waves can be evaluated. The path of each wave is different as seen in Fig. 2; the $N$-th wave travels an extra path of $(2 N-2) \cdot L$ meters with respect to the first transmitted wave. Therefore, if the order of the last wave above the noise level is found, the additional distance travelled by that wave can be obtained and therefore the time gate limits can be calculated. Thus, the attenuation, additional distance, noise level and spatial resolution of the measurement setup are needed. 


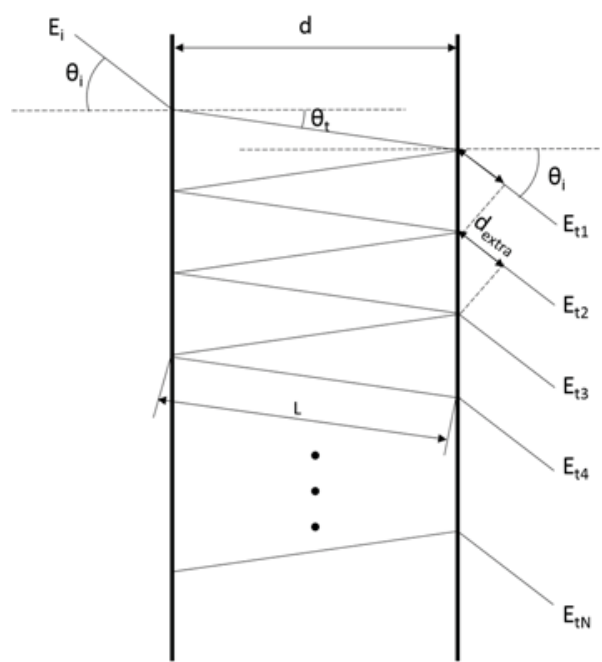

Fig. 2 - scheme of the internal reflections and the transmitted waves

In Table I the attenuation and additional distance for each wave with $N \geq 2$ is shown for the measurement setup. Fig. 3.a) and 3.b) shows the impulse response in the time domain corresponding to the free space situation and when the slab material is present. The main peak when the material is present, which corresponds to the power of $E_{t 1}$, has a relative received power of $-26.81 \mathrm{~dB}$. The noise level is around -80 $\mathrm{dB}$; therefore, every transmitted wave attenuated more than 50 $\mathrm{dB}$ is below the noise. As seen in Table I, the third transmitted wave is attenuated $53 \mathrm{~dB}$, so this wave has a power similar to the noise level; the fourth wave is below the noise floor. The selection of a time gate that includes waves with orders larger than four it is not worth; such time gate only would include multipath components above the noise level and not the desired transmitted waves. Considering that the time resolution of the system is $\Delta \tau=1 / B W=16.67 n s$, which is equivalent to a spatial resolution of $5 \mathrm{~cm}$., a number of samples of 4 is enough to select the transmitted waves from order 1 to 4 . To preserve the symmetry, four samples are taken before and after the main peak as seen in Fig. 3 a) and b).

TABLE I. ATTENUATION AND ADDITIONAL DISTANCE WITH RESPECT $\mathrm{E}_{\mathrm{T} 1}$. ASSOCIATED NUMBER OF SAMPLES INSIDE THE TIME GATE.

\begin{tabular}{|c|c|c|c|}
\hline \multirow{2}{*}{$\begin{array}{c}\text { Comp. } \\
\text { order }\end{array}$} & \multicolumn{3}{|c|}{ Material and setup data: } \\
\cline { 2 - 4 } & $d=3 \mathrm{~cm} ; \varepsilon_{r}=2.4$ (FP estimation); $\theta_{i}=90^{\circ}$. \\
\hline 2 & 26.67 & 0.06 & 2 \\
\hline 3 & 53.34 & 0.12 & 3 \\
\hline 4 & 80.01 & 0.18 & 4 \\
\hline 5 & 106.68 & 0.24 & 5 \\
\hline
\end{tabular}

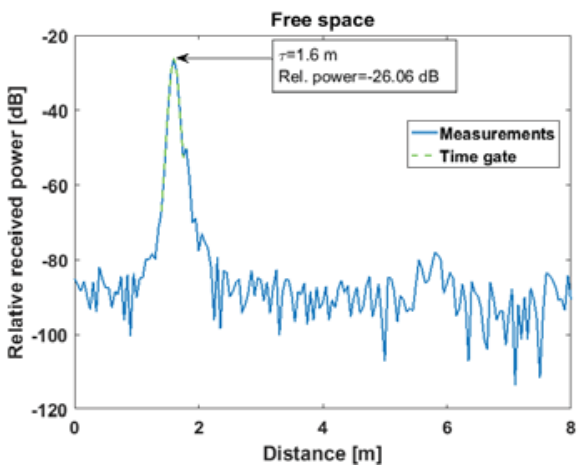

a)

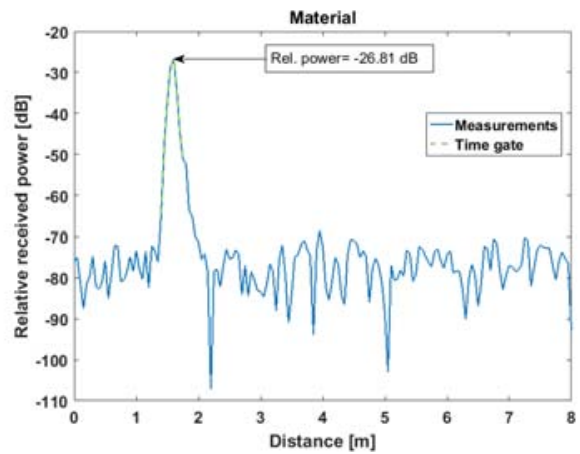

b)

Fig. 3 - Impulse response without a) and with material b) in the transmission measurement. The Hanning window was used. The relative received power of the main peak is shown. The time gate corresponding to 4 samples is also depicted.

Once the time gate size is selected the transmission coefficient can be estimated. The impulse responses are padded with zeros in those samples outside the time gate. Then, the frequency responses are obtained by using the direct Fourier transform. The Hanning window is used in inverse Fourier transform to reduce the sidelobes. The quotient between the frequency responses is the transmission coefficient. To avoid the edge effects produced by the Hanning window application, the bandwidth in the transmission coefficient estimation is limited to $9-13 \mathrm{GHz}$.

The effect of the size of the time gate on the transmission coefficient is seen in Fig. 4. If the size is too low (three samples inside the time gate), the transmission coefficient exhibits irregular oscillations. This behavior is also found if the number of samples is larger than five; the larger the number of samples the larger the undesired oscillations because more and more multipath components fall inside the time gate. Thus, a four samples time gate yields a transmission coefficient which shows a ripple due to the internal reflections and at the same time removes the multipath noise. As seen in the next section, the transmission coefficient obtained using the time gating technique is similar to the coefficient found when a dedicated filter procedure is applied. 


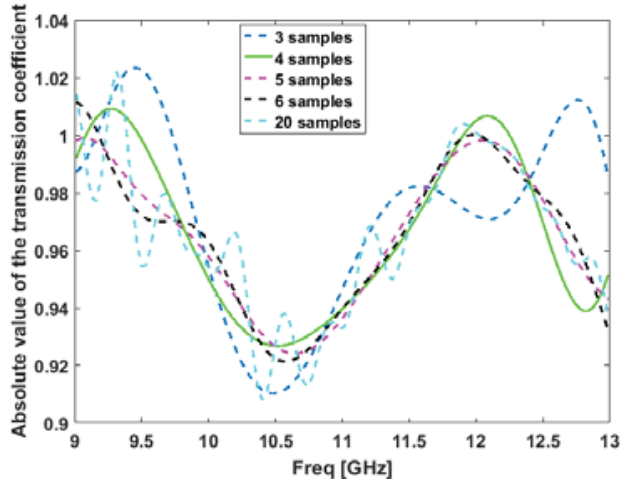

Fig. 4 - Transmission coefficient for different sizes for the time gate.

\section{MEASUREMENT RESULTS}

\section{A. Results}

As mentioned above, the chosen heuristic approach is to compare the transmission coefficient model, computed for different values of sigma, with the measured transmission coefficient, in the considered $8-14 \mathrm{GHz}$ band.

To compute an approximation of sigma, an iterative algorithm was used to find that $\sigma^{\text {opt }}$ that yields the best transmission coefficient's fit of the measured data to the considered transmission model in terms of RSME. The measured transmission coefficient has been previously filtered through the time gating technique described above, in order to remove multipath components' noise. The algorithm operates as follows: an initial range for sigma is given as an input to the algorithm $\left(\sigma^{0}=[0,0.1] \mathrm{S} / \mathrm{m}\right)$; then, at $k$-th iteration, it finds the value of sigma which gives the minimum RMSE in that range $\left(\sigma^{o p t, k}\right)$; finally, it performs a zoom around that value, taking the two immediately adjacent values as the bounds for the next iteration of the algorithm and repeats the procedure. In each iteration, 10 uniformly, distributed values of sigma are taken. The algorithm stops after a maximum number of iterations, which is set to 500 , or when a fixed value of precision (the difference between the upper bound and the lower bound of the range) is reached, set to $10^{-15}$.

Fig. 5 shows the fitting of the measured paraffin transmission coefficient with simulations for 10 values of sigma, at the third iteration.

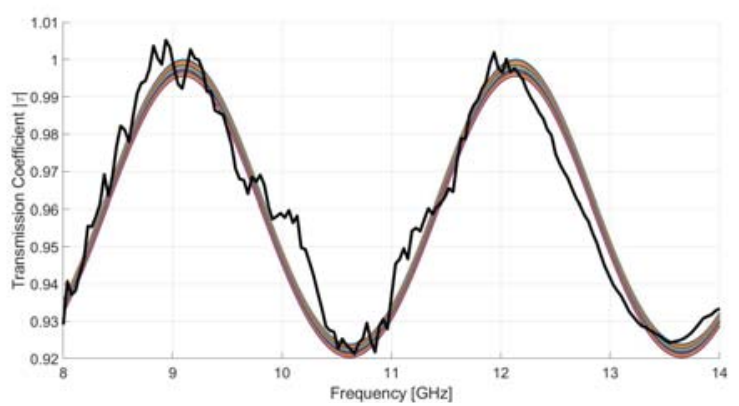

Fig. 5 - Measured (black) and simulated (coloured) transmission coefficient for $\boldsymbol{\sigma}=\left[\mathbf{0}, \mathbf{1 0}^{-3}\right] \mathrm{S} / \mathrm{m}$
In Fig. 6 the corresponding RMSE is plotted as a function of sigma.

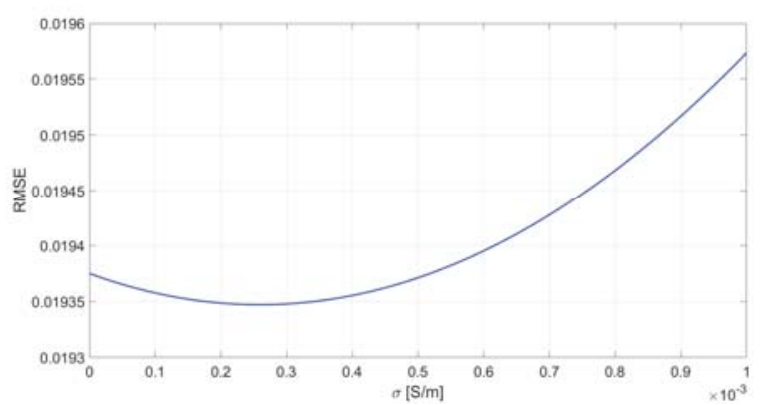

Fig. 6 - RMSE as a function of sigma at the third iteration. Sigma ranges from 0 to $\mathbf{1 0}^{-3} \mathrm{~S} / \mathrm{m}$

The algorithm stops after 21 iterations $(1.2895 \mathrm{~s})$, giving a value of $\sigma^{\text {opt }}=2.592 \cdot 10^{-4} \mathrm{~S} / \mathrm{m}$. In Fig. 7, the measured and filtered transmission coefficient are reported together with the model computed according the achieved optimum value of sigma $\sigma^{\text {opt }}$.

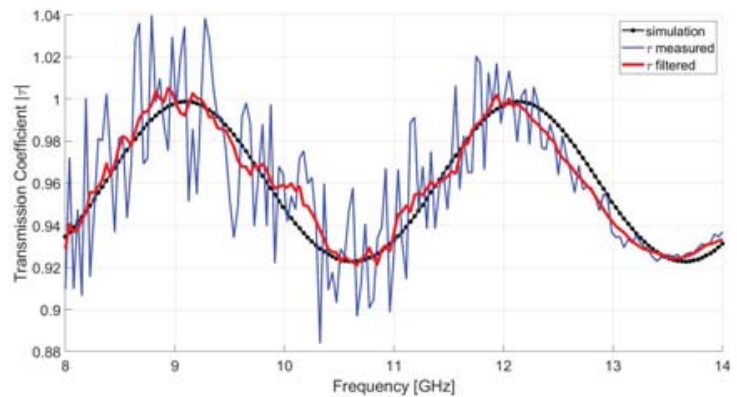

Fig. 7 - Measured (blue) and filtered (red) transmission coefficient for the paraffin slab as a function of frequency. Simulated transmission coefficient (black) computed with the best value of sigma

The achieved optimal sigma leads to a tangent loss $\tan \delta=$ $1.89 \cdot 10^{-4}$ which is in line with the literature [7], [10].

Such results are to be taken as mean values in the considered (wide) frequency band.

The above results are then compared and assessed with those of an open-ended coaxial probe-based method, which are shown in Fig. 8. 


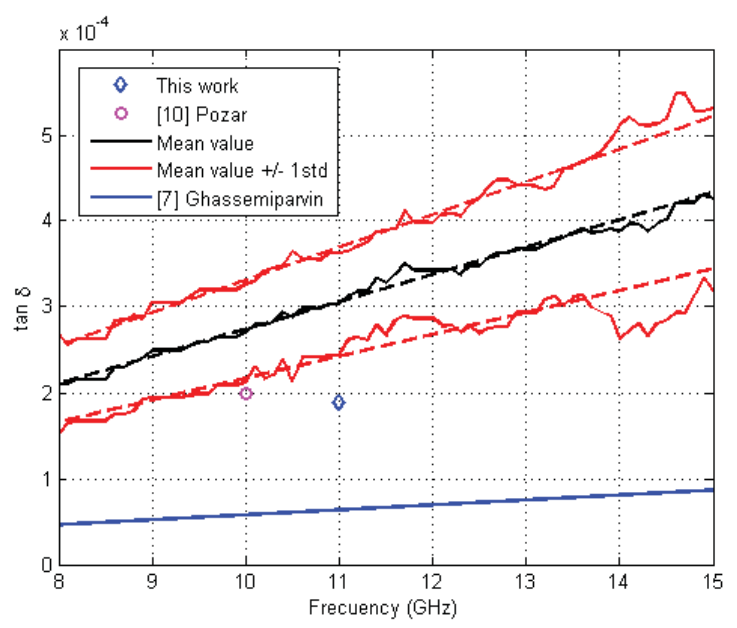

Fig. 8 - Measured (continuous line) and first order fitted (dashed line) results for 9 samples of paraffine wax with an open-ended coaxial probe.

In Fig. 8 a comparison between different methods is shown: the value obtained in this work in a blue diamond; results from [10] in a pink circle; mean and $-1 /+1$ standard deviation of the measured material (averaged 9 samples) using a commercial open-ended coaxial probe DAK 3.5 (Dielectric Assessment Kit) from SPEAG Switzerland in black and red (continuous measured, discontinuous first order fit); and results from [7] in blue. From these results we observed that our method is in consonant with [10], and between two different techniques.

It also turned out that sigma has a very small value, leading to deduce that paraffin is a very low loss material. For this reason, it may take place that the measured transmission coefficient goes higher than 1 in some points, due to noise and impairments of the equipment.

\section{CONCLUSIONS}

A method to extract the imaginary part of the complex permittivity of a material's slab is shown in this work, based on a least-square optimization method and on the proper use of time-gating [3] to eliminate environment multipath or, if viable case, to cut off the FP resonance from the transmission measurement. Results of the proposed technique in this work are in good agreement with the open-ended coaxial probe assessment and with literature.

The assessment of the method with other construction materials and frequencies will be presented in the final version of this paper. In the future, a comprehensive list of materials will be considered in several 1-100 GHz frequency bands for $5 \mathrm{G}$ and beyond applications.

\section{ACKNOWLEDGEMENT}

This work was supported by the Spain Ministerio de Economía, Industria y Competitividad under grant TEC201678028-C3-2-P. The authors would like to thank Prof. Alberto Santarelli and Dr. Corrado Florian of the DEI Department,
University of Bologna, for their help in performing the VNA measurements

\section{REFERENCES}

[1] S. Salous et al., "Millimeter-wave propagation: Characterization and modeling toward 5fth-generation systems. [Wireless Corner]," IEEE Antennas and Propag. Mag., vol. 58, no. 6, pp. 115-127, Dec. 2016.

[2] V. Degli-Esposti, M. Zoli, E. M. Vitucci, F.Fuschini, M. Barbiroli, J Chen, "A Method for the Electromagnetic Characterization of Construction Materials based on Fabry-Pérot Resonance," IEEE Access, pp 24938 - 24943 Vol. 5, 2017.

[3] M. Martinez-Ingles, J. Rodriguez, J. Molina-Garcia-Pardo, J. PascualGarcia and L. Juan-Llacer, "Comparison of a UTD-PO Formulation for Multiple-Plateau Diffraction With Measurements at $62 \mathrm{GHz}$," in IEEE Transactions on Antennas and Propagation, vol. 61, no. 2, pp. 10001003, Feb. 2013.

[4] M. A. Stuchly, M. M. Brady, S. S. Stuchly, and G. Gajda, "Equivalent circuit of an open-ended coaxial line in a lossy dielectric," IEEE Trans. Instrum. Meas., vol. IM-31, no. 2, pp. 116-119, Jun. 1982.

[5] G. Durgin, T. S. Rappaport, H. Xu, "Measurements and Models for Radio Path Loss and Penetration Loss In and Around Homes and Trees at $5.85 \mathrm{GHz}$ ", IEEE Trans. on Communications, vol. 46, No. 11, November 1998

[6] D. Plets, W. Joseph, L. Verloock, L. Martens, H. Gauderis, E. Deventer, "Extensive Penetration Loss Measurement and Models for Different Building Types for DVB-H in the UHF Band", IEEE Trans. On Broadcasting, vol. 55, No. 2, June 2009, pp. 213-222

[7] B. Ghassemiparvin and N Ghalichechian, "Complex Permittivity Measurement of Paraffin Phase-Change Material at $26 \mathrm{GHz}-1.1 \mathrm{THz}$ Using Time-Domain Spectroscopy", International Journal of Infrared and Millimeter Waves, Vol.40, February 2019, pp. 210-218.

[8] S. Manzari, A. A. Babar, L. Ukkonen, Atef Z. Elsherbeni, G. Marrocco, and L. Sydanheimo, "Performance Analysis of Pure Paraffin Wax as RFID Tag Substrate", MICROWAVE AND OPTICAL TECHNOLOGY LETTERS / Vol. 54, No. 2, February 2012 DOI 10.1002/mop.

[9] W. D. Burnside, "High frequency scattering by a thin lossless dielectric slab," IEEE Transaction on antennas and propagation, Vol. 31, pp. 104110, January 1983.

[10] David. M. Pozar, "Microwave Engineering", 4th ed. John Wiley \& Sons, Inc., 2012, pp. 719-720 